\title{
Violence: heightened brain attentional network response is selectively muted in Down syndrome
}

\author{
Jeffrey S. Anderson 1,2,3,4*, Scott M. Treiman ${ }^{3}$, Michael A. Ferguson ${ }^{4}$, Jared A. Nielsen², Jamie O. Edgin ${ }^{5}$, Li Dai ${ }^{6}$, \\ Guido Gerig ${ }^{3,4,7}$ and Julie R. Korenberg ${ }^{2,3,6}$
}

\begin{abstract}
Background: The ability to recognize and respond appropriately to threat is critical to survival, and the neural substrates subserving attention to threat may be probed using depictions of media violence. Whether neural responses to potential threat differ in Down syndrome is not known.

Methods: We performed functional MRI scans of 15 adolescent and adult Down syndrome and 14 typically developing individuals, group matched by age and gender, during 50 min of passive cartoon viewing. Brain activation to auditory and visual features, violence, and presence of the protagonist and antagonist were compared across cartoon segments. fMRI signal from the brain's dorsal attention network was compared to thematic and violent events within the cartoons between Down syndrome and control samples.

Results: We found that in typical development, the brain's dorsal attention network was most active during violent scenes in the cartoons and that this was significantly and specifically reduced in Down syndrome. When the antagonist was on screen, there was significantly less activation in the left medial temporal lobe of individuals with Down syndrome. As scenes represented greater relative threat, the disparity between attentional brain activation in Down syndrome and control individuals increased. There was a reduction in the temporal autocorrelation of the dorsal attention network, consistent with a shortened attention span in Down syndrome. Individuals with Down syndrome exhibited significantly reduced activation in primary sensory cortices, and such perceptual impairments may constrain their ability to respond to more complex social cues such as violence.

Conclusions: These findings may indicate a relative deficit in emotive perception of violence in Down syndrome, possibly mediated by impaired sensory perception and hypoactivation of medial temporal structures in response to threats, with relative preservation of activity in pro-social brain regions. These findings indicate that specific genetic differences associated with Down syndrome can modulate the brain's response to violence and other complex emotive ideas.
\end{abstract}

Keywords: fMRI, Violence, Down syndrome, Attention

\section{Background}

Down syndrome is a prevalent intellectual disability syndrome occurring in 9.0 to 11.8 per 10,000 live births [1] associated with intellectual, motor, memory, and language impairments [2]. Despite moderately severe, though variable, cognitive impairments, individuals with Down

\footnotetext{
*Correspondence: andersonjeffs@gmail.com

'Department of Radiology, 1A71 School of Medicine, University of Utah, Salt Lake City, UT 84132, USA

${ }^{2}$ Interdepartmental Program in Neuroscience, University of Utah, Salt Lake City, USA

Full list of author information is available at the end of the article
}

syndrome are often perceived as particularly affectionate and happy [3]. In contrast to other neurodevelopmental conditions such as autism, where social function is a particular deficit, individuals with Down syndrome, as in Williams syndrome, exhibit some relative strengths in social function compared to other neurocognitive domains [4]. However, aspects of social cognition have shown particular impairments, especially emotion recognition, and in particular, the recognition of surprise and fear [5].

Relatively little is known about brain activation differences in individuals with Down syndrome. Two prior task-based studies have been reported using functional MRI in Down syndrome. During passive story listening,

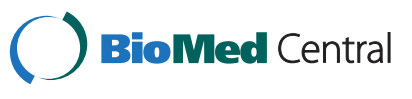


classical language regions showed decreased activation relative to controls in one study [6]. In another study of object recognition, correlations between brain activation and a metric of visuospatial ability were demonstrated in middle and dorsal frontal gyri in Down syndrome but in the occipital and parietal lobes for typically developing individuals [7].

Functional MRI connectivity is also abnormal in Down syndrome. We recently reported that functional connectivity is abnormal in Down syndrome, with generalized increased synchrony between distributed brain networks and deficits in both negatively correlated and long-range positively correlated connections [8]. The finding of between-network hyperconnectivity was also observed independently by Vega and colleagues [9]. Moreover, hyperconnectivity between the anterior temporal and anterior cingulate cortex, in addition to reduced within-network connectivity in the dorsal attention network, was also observed by Pujol and colleagues [10]. These findings contributed to impaired scores on adaptive function testing [10]. In contrast, for a near-infrared spectroscopy study performed on infants with Down syndrome, lower mean connectivity between channels was observed [11].

In our prior report [8], participants watched cartoon audiovisual stimuli for extended periods $(50 \mathrm{~min}$ per participant) in order to minimize participant motion and maintain wakefulness. Yet the cartoons themselves provide a rich substrate for probing social interactions to protagonists, antagonists, and violence. In particular, the Bugs Bunny cartoons viewed contain extensive social interactions among characters as well as stylized depictions of humorous but extreme cartoon violence such as characters falling off cliffs, characters being assaulted with blunt objects, or characters smoking exploding cigars. These types of scenes receive ubiquitous media attention for potentially adverse consequences on aggressivity and mental health development in children given the large amount of time children spend viewing cartoons, yet we found no characterization in the imaging literature of brain responses to such stylized violence in either typically developing individuals or those with developmental syndromes. We examined functional brain activation in response to such stimuli in Down syndrome and in typical development to determine whether regional brain activation patterns could be characterized, as well as whether atypical neural activation might be present that could provide clues to a brain basis for the deficits seen in Down syndrome.

\section{Methods}

\section{Participant characteristics}

Analyses for this report were performed based on the data from 15 individuals with Down syndrome (mean age $20.2 \pm 6.3$, range 14-34, 9 males, 6 females) and 14 typically developing participants (mean age $23.7 \pm 5.9$, range 15-39, 8 males, 6 females), displayed in Table 1 . Three participants with Down syndrome and two control participants were left-handed. All study procedures were performed in compliance with guidelines approved by the University of Utah Institutional Review Board. Informed consent or assent (with guardian consent) was obtained from all participants with Down syndrome and controls.

All participants with Down syndrome, recruited from the community, underwent genotyping to confirm trisomy 21. One participant exhibited genetic mosaicism for Down syndrome but showed facial, behavioral, and cognitive deficits characteristic of Down syndrome. Group differences in activation were not qualitatively different when this subject was excluded. One additional participant with Down syndrome was excluded from all analyses due to excessive motion during the scan and was not included in the total. Control participants were recruited from the community. For both control and participants with Down syndrome, medical history and structured psychiatric interview (DSM-IV) were performed by an experienced physician in neurodevelopmental disorders [12]. No control participants had a history of developmental, learning, cognitive, neurological, or neuropsychiatric Axis I condition. Verbal IQ (VIQ) and performance IQ (PIQ) were measured with the Kaufman Brief Intelligence Test, Second Edition [13]. IQ measurements were performed in a subset (8/14) of control participants (mean 107.6 \pm 12.7 VIQ; $111.7 \pm$ 13.8 PIQ) and 14/15 participants with Down syndrome (53.1 \pm 12.6 VIQ; $44.9 \pm 5.4$ PIQ). One participant with Down syndrome was not able to complete IQ testing. We note that our control cohort exhibits slightly higher IQ than the general population. Six of the participants with Down syndrome were taking no medications at the time of the scan. Other participants were taking levothyroxine $(n=5)$, microgestin $(n=1)$, enalapril $(n=1)$, omeprazole $(n=1)$, lansoprazole $(n=1)$, fluticasone proprionate $(n=1)$, insulin $(n=2)$, albuterol $(n=2)$, and sertraline $(n=1)$.

As a template of the attention control network, comprising both dorsal attention and ventral attention networks, we examined data from 1011 subjects from

Table 1 Demographics of study participants

\begin{tabular}{llll}
\hline & Age & VIQ & PIQ \\
\hline Down syndrome $(n)$ & $15(9 \mathrm{M}, 6 \mathrm{~F})$ & 14 & 14 \\
DS mean \pm s.d. & $20.2 \pm 6.3(14-34)$ & $53.1 \pm 12.6$ & $44.9 \pm 5.4$ \\
Control $(n)$ & $14(8 \mathrm{M}, 6 \mathrm{~F})$ & 8 & 8 \\
Control mean \pm s.d. & $23.7 \pm 5.9(15-39)$ & $107.6 \pm 12.7$ & $111.7 \pm 13.8$ \\
$p$-Value (two-tailed $t$ test) & 0.14 & $5.1 \times 10^{-9}$ & $5.0 \times 10^{-13}$ \\
\hline
\end{tabular}


publicly available datasets released with the open-access 1000 Functional Connectomes Project (http://fcon_1000. projects.nitrc.org) in which resting-state functional magnetic resonance imaging (fMRI) scans have been aggregated from 28 sites [14] as well as typically developing subjects from the ADHD 200 project from the International Neuroimaging Data-sharing Initiative (fcon_1000. projects.nitrc.org/indi/adhd200/index.html) including 8 sites [15]. Data processing and subject selection has been previously described [16]. We calculated mean functional correlation to four $5-\mathrm{mm}$ radius ROIs in the bilateral anterior insula (left anterior insula $x=-44, y=8$, $z=4$; right anterior insula $x=48, y=10, z=0$ ) and intraparietal sulcus (left IPS $x=-48, y=-38, z=51$; right IPS $x$ $=44, \quad y=-38, z=49$ ).

\section{Image acquisition}

Images were acquired on Siemens 3 Tesla Trio scanner with 12-channel head coil. The scanning protocol consisted of initial $1 \mathrm{~mm}$ isotropic MPRAGE acquisition for an anatomic Table 1. BOLD echoplanar images $(\mathrm{TR}=2.0$ $\mathrm{s}, \mathrm{TE}=28 \mathrm{~ms}$, GRAPPA parallel acquisition with acceleration factor $=2$, 40 slices at $3 \mathrm{~mm}$ slice thickness, $64 \times 64$ matrix) were obtained while viewing Bugs Bunny cartoons (Looney Tunes Golden Collection Volume 1, Warner Home Video) [17]. These consisted of 10 5-min clips, none of which contained a complete cartoon. The following 10 clips were used, beginning at the opening credits for each clip: "Baseball Bugs", "High Diving Hare", "Bully for Bugs", "What's Up Doc", "Ballot Box Bunny", "Rabbit of Seville", "Wabbit Twouble", "Rabbit's Kin", "LongHaired Hare", and "Rabbit Seasoning". Both auditory and visual components of the video were presented. Subjectively, both control and participants with Down syndrome appeared to tolerate the cartoons well and remained awake during the stimuli as observed by live video feed of the participants' eyes during examination. Data was obtained during 10 five-minute cartoons for each participant, presented in the same order for each participant. The stimulus computer was synchronized to the onset of the first BOLD image via fiber optic pulse emitted by the scanner for reproducible, precise onset timing. Images were presented through LCD projection onto a screen positioned within the bore of the scanner and viewed via a mirror positioned on the top of the head coil. Auditory stimuli were presented through MRI-compatible headphones (Avotec).

\section{fMRI preprocessing}

Offline preprocessing was performed in MATLAB (Mathworks, Natick, MA, USA) using SPM8 (Wellcome Trust, London, UK) software. Initial slice timing correction was performed to adjust for interleaved slice acquisition. All images were motion corrected using the realign procedure. BOLD images were coregistered to the MPRAGE anatomic image sequence for each participant. All images were normalized to the MNI template brain (T1.nii in SPM8), with manual inspection of appropriate normalization in all participants. Spatial smoothing was performed with $8 \mathrm{~mm}$ FWHM kernel.

\section{Statistical analysis}

Time courses for all 10 cartoon clips were obtained prior to analysis by 2 observers blinded to study design and data consisting of second-by-second identification of whether (1) the protagonist was on screen $(51.3 \%$ of the cartoons), (2) the antagonist was on screen ( $47.3 \%$ of the cartoons), and (3) a violent act or accident likely to result in bodily harm was taking place against any character $(4.2 \%$ of the cartoons). Interrater reliability was assessed using Cohen's kappa (MATLAB) and found to show $\mathrm{K}=0.65$ for protagonist on screen (substantial agreement), 0.55 for villain on screen (moderate agreement), and 0.49 for violent scenes (moderate agreement). Only scenes scored by both observers were used for subsequent analysis.

A general linear model was used to evaluate for brain activation associated with epochs when the protagonist was on screen, antagonist was on screen, and for violent scenes, with multiple regressors including six head motion parameters for each subject, auditory volume, and visual complexity of the video scenes. Auditory volume was assessed by measuring the waveform of the sound presented during the movie and measuring rootmean-square of the signal for timepoints within each brain volume acquired. This time series was convolved with a hemodynamic response function (spm_hrf [2]) and used as a regressor. Visual complexity consisted of the average root-mean-square difference in red, green, and blue pixel intensity from frame to frame, averaged across all pixels and for all frames within each brain volume acquisition. This time series was also convolved with a hemodynamic response function and used as a regressor in the model.

This model generated contrasts in a first level eventrelated paradigm in each participant, with all 10 cartoons concatenated for each participant. Second-level group analysis consisted of two-tailed $t$ tests for each group independently and for DS > control and control $>$ DS, with significant clusters requiring acceptable false discovery rate $q<0.05$ to account for multiple comparisons.

\section{Attentional network analysis}

To analyze fluctuations in activation in brain attentional regions, we identified a priori a set of voxels comprising the brain's dorsal attention network from a prior report in the literature [18]. These voxels were used as a restriction mask and average time series for these voxels 
was calculated for each participant in each cartoon. For each participant and each cartoon, relative activation was obtained by linear detrending, subtracting the mean and dividing by the standard deviation of the BOLD signal. For the mean dorsal attention network activation in each participant sample for each cartoon, $95 \%$ confidence intervals were calculated. Additionally, the temporal autocorrelogram was calculated for each participant's time series in each cartoon for \pm 3 lags, with Fisher transformation of the autocorrelograms to improve normality. Average autocorrelograms were obtained for each participant (averaged across all cartoons) and for each cartoon in each participant sample (Down syndrome, control). Averaged measurements were converted back to correlation measurements by an inverse Fisher transformation for display. Full-width half maximum measurements were obtained by performing linear interpolation of datapoints in the averaged autocorrelograms, and a two-tailed $t$ test was performed between groups of participants on the FWHM for the autocorrelograms.

\section{Results}

Activation maps for the five event-related contrasts studied (antagonist on screen, protagonist on screen, auditory, visual, and violence) are shown for Down syndrome and control samples in Fig. 1a, thresholded at $p<0.001$, cluster corrected by false discovery rate, for display. Significant activation is more extensive for four of the contrasts in the control group than in the Down syndrome group but not for the protagonist on screen contrast (top row), in which the two samples show similar activation. Auditory and visual contrasts activate the primary auditory and primary and secondary visual cortex, respectively, with some activation of the visual cortex observed in controls for the auditory contrast as well. For protagonist on screen, antagonist on screen, and violence contrasts, the activated regions closely conformed to the expected location of the dorsal attention network, including bilateral intraparietal sulcus, frontal eye fields, and middle temporal cortex. This network is illustrated in Fig. 1b, derived from 1011 subjects from the 1000 Functional Connectomes dataset [14]. Activation of the dorsal attention network was spatially homologous between the two groups, and given that the primary contrasts associated with protagonist, antagonist, and violence recapitulated this network, with no significant activation of amydalar, orbitofrontal, insular or other emotive brain regions, the dorsal attention network was used as the primary metric in subsequent analyses.

To directly measure between-sample differences in activation, a two-tailed $t$ test was performed for each of the five contrasts, and significant differences were observed for four of the contrasts, auditory, visual, violence, and antagonist on screen, with significant clusters reported in Table 2 and shown in Fig. 2 for each contrast. For the antagonist on screen contrast, control participants showed greater activation in the left entorhinal cortex as well as bilateral frontal eye fields, middle temporal, cerebellar hemisphere, and supplementary motor area. For the auditory contrast, control participants showed greater activation of the left primary auditory cortex. For the visual contrast, control subjects showed greater activation of most of the visual cortex, including striate and extrastriate cortex. For the violence contrast, control participants showed greater activation throughout a distributed set of brain regions closely aligned with the brain's dorsal attention network. Participants with Down syndrome showed significantly greater activation of bilateral primary auditory cortex during violent scenes. None of the other contrasts showed any clusters with significantly higher activation in Down syndrome.

We next attempted to determine whether specific features of the cartoons resulted in the apparent decreased attentional network activation in Down syndrome. By creating a restriction mask from voxels in the dorsal attention network from the literature, we obtained average time series from this network for each participant, with mean time courses shown in Figs. 3 and 4 for Down syndrome and control samples. For timepoints where the $95 \%$ confidence intervals were not overlapping and which showed the greatest differences between groups in relative dorsal attention network activation, screenshots display the content of the cartoons. Six seconds was subtracted from the timepoint in each case to correct for the effects of the hemodynamic response function. For all timepoints where $95 \%$ confidence intervals did not overlap, a brief description of the scene is tabulated in Additional file 1: Tables S1 and S2.

Timepoints for which the control sample showed higher activation frequently correspond to activation peaks and occur at among the most violent scenes in the cartoons. In contrast, timepoints where participants with Down syndrome had higher activation are most typically at points where control participants show low activation, such as during opening credits (7 of 10 cartoons significantly higher in Down syndrome), or during scenes devoid of violence, such as Bugs Bunny dancing, closeup facial images of protagonists, or outdoor scenes. Of scenes where $95 \%$ confidence intervals for mean dorsal attention network signal did not overlap between the two groups, 20 out of 37 of the scenes where control participants had higher signal involved violence, while only 1 out of 36 of the scenes where participants with Down syndrome had higher signal involved violence.

Only two cases of romantic or sexually themed content were present in the 10 cartoons showing differences in attentional activation between the groups: Bugs Bunny 


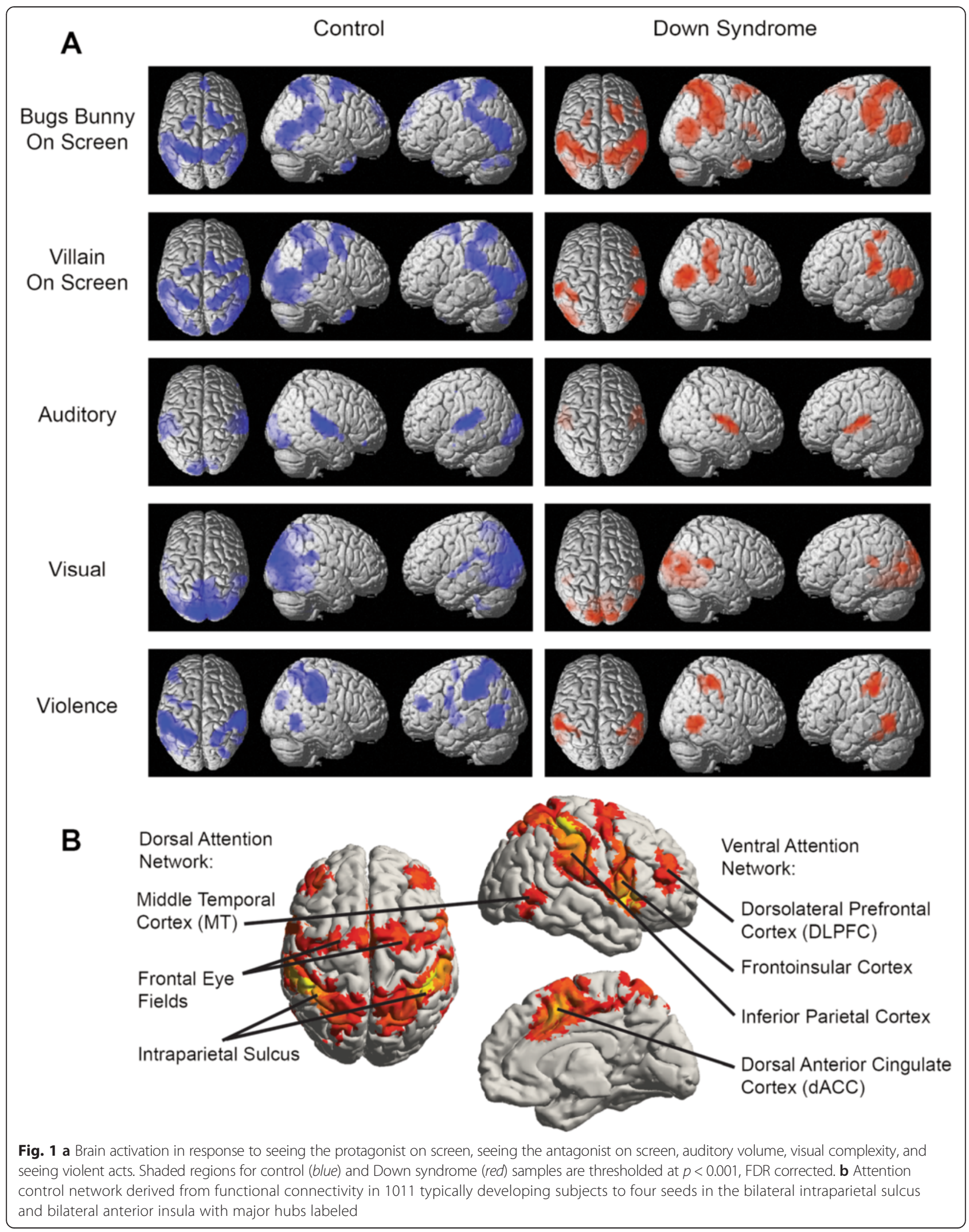


Table 2 Clusters showing significant difference in activation between control and Down syndrome groups. All clusters showed higher activation in control sample. $q$ values show cluster-corrected false discovery rate. T-statistics show peak voxelwise results

\begin{tabular}{|c|c|c|c|c|}
\hline Contrast & Region & MNI coordinates & Cluster q-value (FDR) & Number of voxels \\
\hline \multicolumn{5}{|l|}{ Control > DS } \\
\hline Antagonist on screen & Left intraparietal sulcus & $(-21-5567)$ & 0.00000009 & 284 \\
\hline Antagonist on screen & Right frontal eye field & $(18-1055)$ & 0.000002 & 383 \\
\hline Antagonist on screen & Left frontal eye field & $(-24-1061)$ & 0.008 & 80 \\
\hline Antagonist on screen & Left middle temporal & $(-36-644)$ & 0.000009 & 230 \\
\hline Antagonist on screen & Left parahippocampal & $(-24-22-23)$ & 0.011 & 66 \\
\hline Antagonist on screen & Left middle occipital & $(-48-821)$ & 0.016 & 57 \\
\hline Antagonist on screen & Left cerebellum & $(-24-61-17)$ & 0.008 & 76 \\
\hline Antagonist on screen & Right superior occipital & $(18-9134)$ & 0.038 & 43 \\
\hline Antagonist on screen & Left cerebellum & $(-12-73-47)$ & 0.008 & 74 \\
\hline Antagonist on screen & Right supramarginal & $(57-2831)$ & 0.06 & 34 \\
\hline Antagonist on screen & Right intraparietal sulcus & $(18-6161)$ & 0.045 & 39 \\
\hline Auditory & Left superior temporal & $(63-164)$ & 0.001 & 117 \\
\hline Visual & Bilateral occipital & $(18-7637)$ & $3.7 \times 10^{-28}$ & 2428 \\
\hline Violence & Left intraparietal sulcus & $(-18-5573)$ & 0.000008 & 229 \\
\hline Violence & Left posterior insula & $(-36-131)$ & 0.04 & 44 \\
\hline Violence & Left supramarginal & $(-54-3131)$ & 0.05 & 33 \\
\hline Violence & Left frontal eye field & $(-27-755)$ & 0.04 & 43 \\
\hline Violence & Left middle temporal & $(-39-581)$ & 0.04 & 40 \\
\hline \multicolumn{5}{|l|}{ DS > control } \\
\hline Violence & Right superior temporal & $(63-194)$ & 0.0001 & 153 \\
\hline Violence & Left superior temporal & $(-57-404)$ & 0.01 & 54 \\
\hline
\end{tabular}

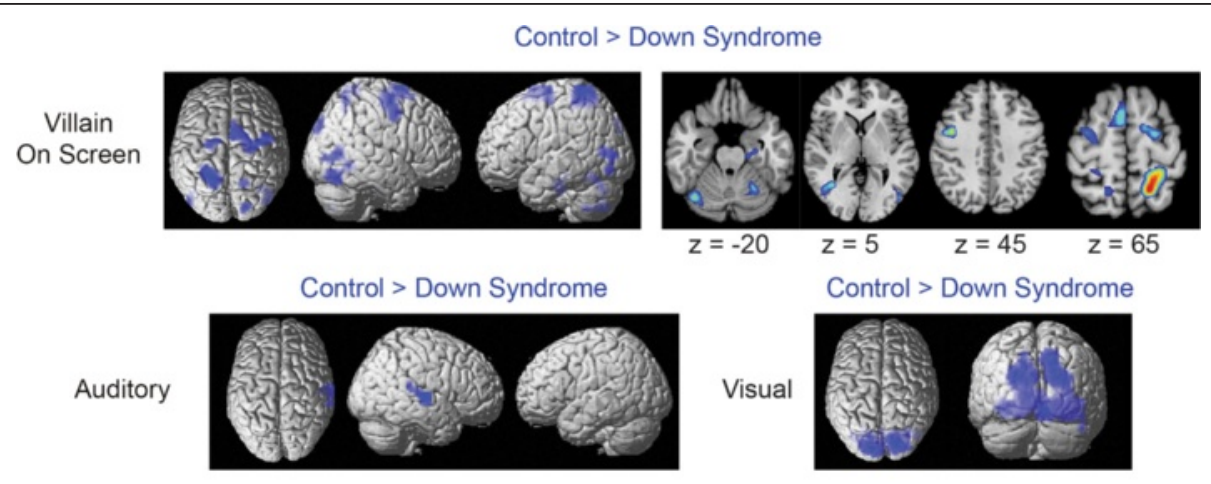

Control > Down Syndrome Down Syndrome > Control

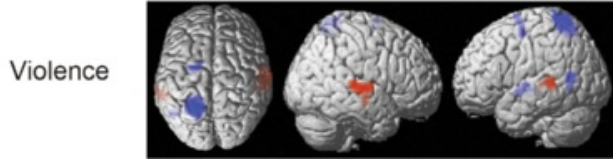

Fig. 2 Differences in brain activation between Down syndrome and control samples. Brain regions showing greater activation for control participants are shown in rendered images (upper left) and slices (upper right) in response to seeing the antagonist on screen, with slice locations in radiological format given by MNI z-coordinate under the image. Brain regions showing significantly greater activation for control participants than for participants with Down syndrome are shown in the center in response to auditory volume and visual complexity of the video cartoons. Images showing greater activation for control individuals (blue) and individuals with Down syndrome (red) in response to violent scenes are shown in the image. All images are thresholded at $p<0.001$, FDR corrected 


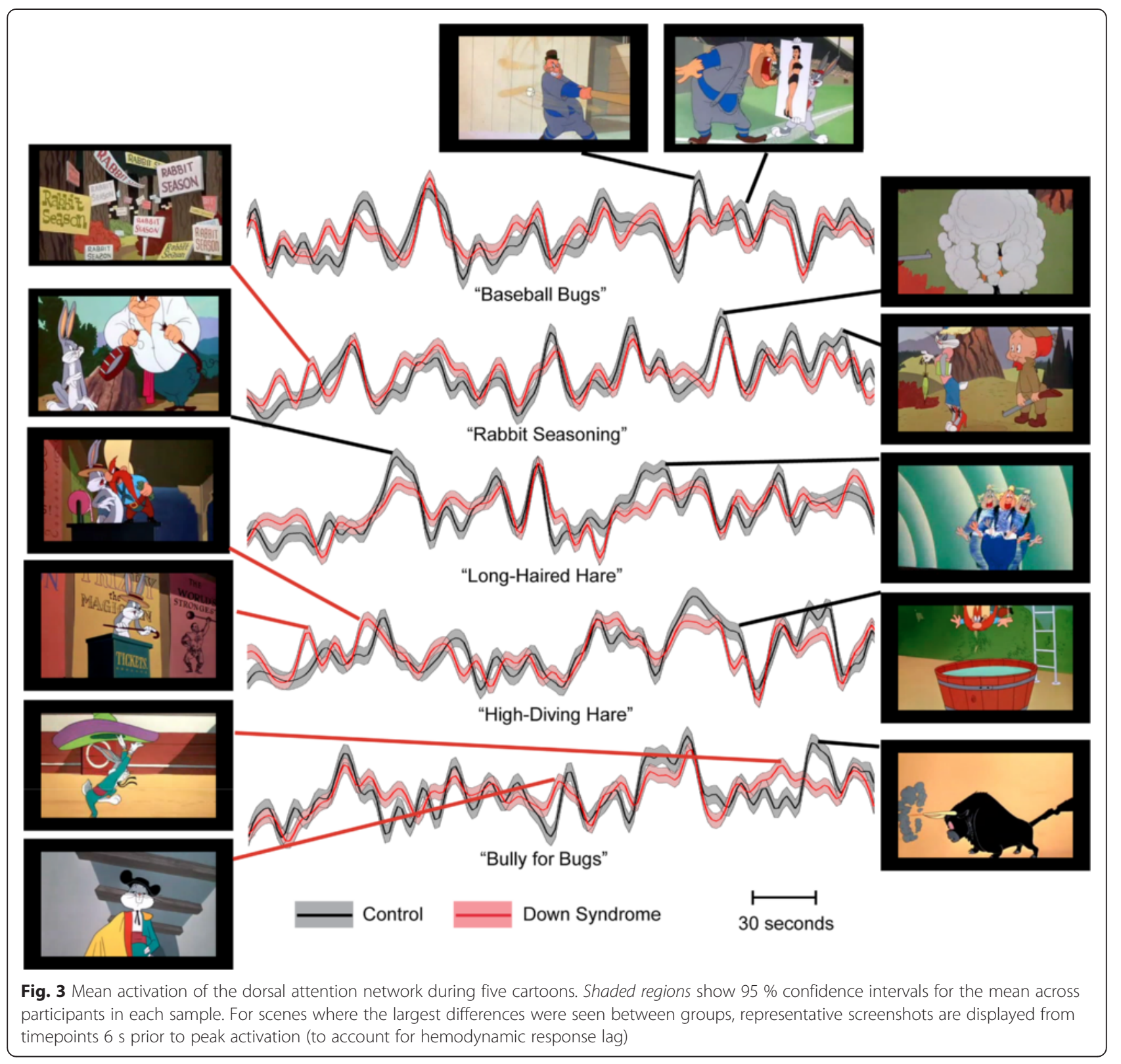

holding a pinup image to distract the catcher in a baseball game and Bugs Bunny dressing as a female to lure Elmer Fudd. In both cases, control participants showed significantly higher activation.

Comparisons of temporal autocorrelation during the cartoons are shown in Fig. 5. For all 10 cartoons, there was markedly narrower autocorrelation for participants with Down syndrome than for control participants. When evaluating individual participants, the full-width half maximum of autocorrelograms was significantly greater for the control group (mean control $2.60 \pm 0.41$ s.d., Down Syndrome $1.93 \pm 0.26$ s.d., $p=0.000016$ ). This finding would be consistent with more idiosyncratic activation of the dorsal attention network or shorter periods of sustained activation within the network.

When activated voxels are tallied for control and individuals with Down syndrome, a progression is seen with increasing disparity between the two groups as the relative threat of the stimuli increases from seeing Bugs Bunny to seeing the antagonist to actual violence within the scene. This is illustrated quantitatively and graphically in Fig. 6.

\section{Discussion}

In a sample of 15 Down syndrome and 14 typically developing control participants, we found that brain 


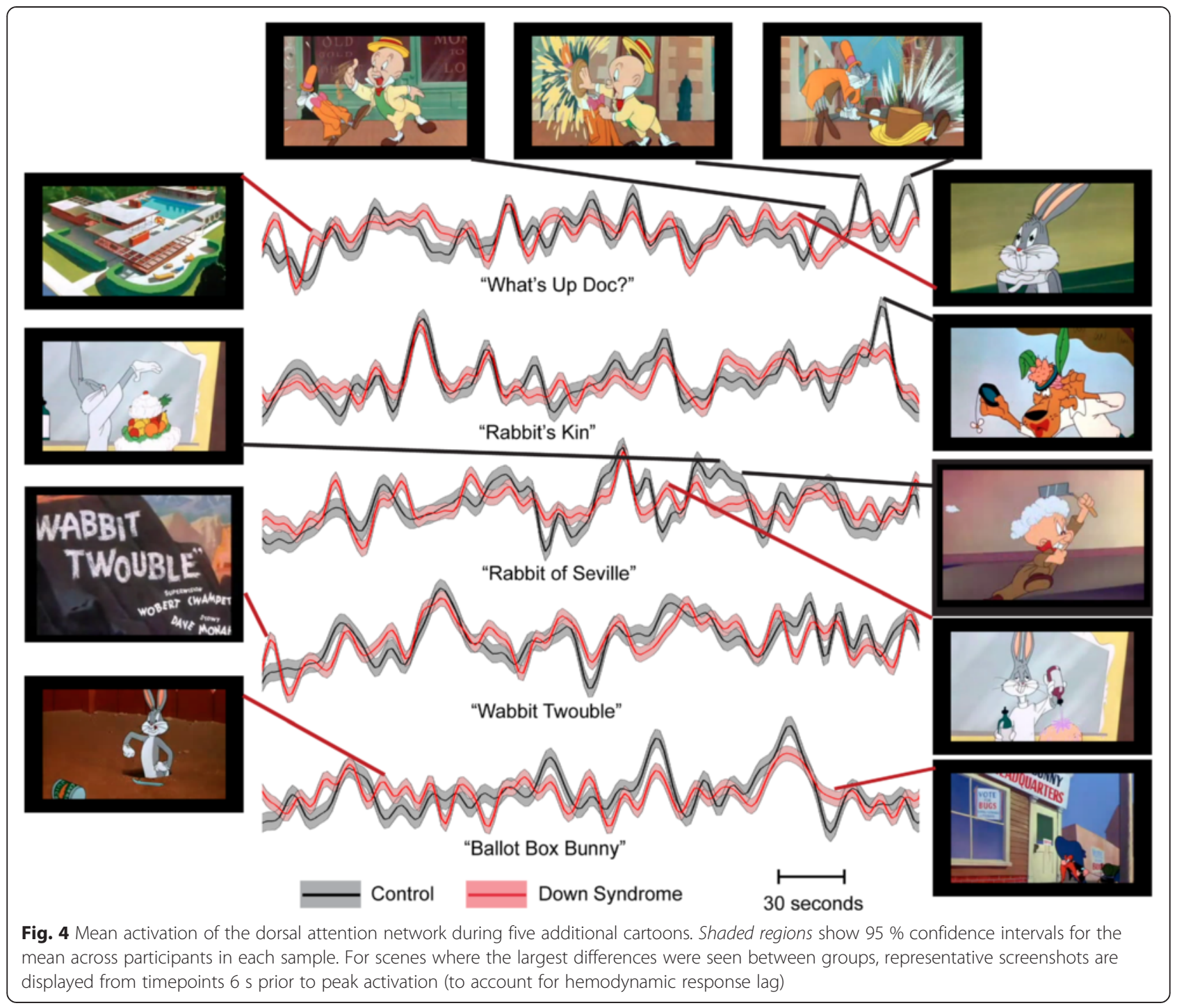

responses to passive cartoon viewing were largely similar between the groups, with similar brain regions that were activated despite marked functional impairment of the Down syndrome group. Nevertheless, brain activation to cartoon stimuli was significantly weaker in brain attentional regions in response to primary auditory and visual sensory stimulation, scenes involving the villain, and violent scenes in Down syndrome. The decreased responses to violence were specific to the extent that violent scenes could be predicted by differences in activation from moment to moment in the brain's dorsal attention network. Brain responses to an "enemy" or "antagonist" present on screen were particularly low in Down syndrome in the dorsal attention network and left medial temporal lobe. These data provide some of the first evidence regarding the brain networks that may correspond to altered cognition in certain social cognitive situations in those with Down syndrome, such as those including negative emotions or violence. Dorsal attention network activation showed reduced temporal autocorrelation in Down syndrome, possibly consistent with shortened attention span or inability to sustain attentional activation over extended periods of time.

The brain's dorsal attention network has been the subject of recent study in numerous reports. These regions, consisting of the bilateral intraparietal sulcus, frontal eye fields, and middle temporal (MT) regions, have been strongly implicated as the locus for attention to external stimuli [19] and show activation when an individual attends to audiovisual stimuli $[15,20]$. This network is organized internally by sensory modality [21] and has shown a mutually anticorrelated relationship with the brain's internal stimulus processing network, the default mode network $[22,23]$. It is important to note that the brain regions of the dorsal attention network are polyfunctional and involve complex polymodal association 


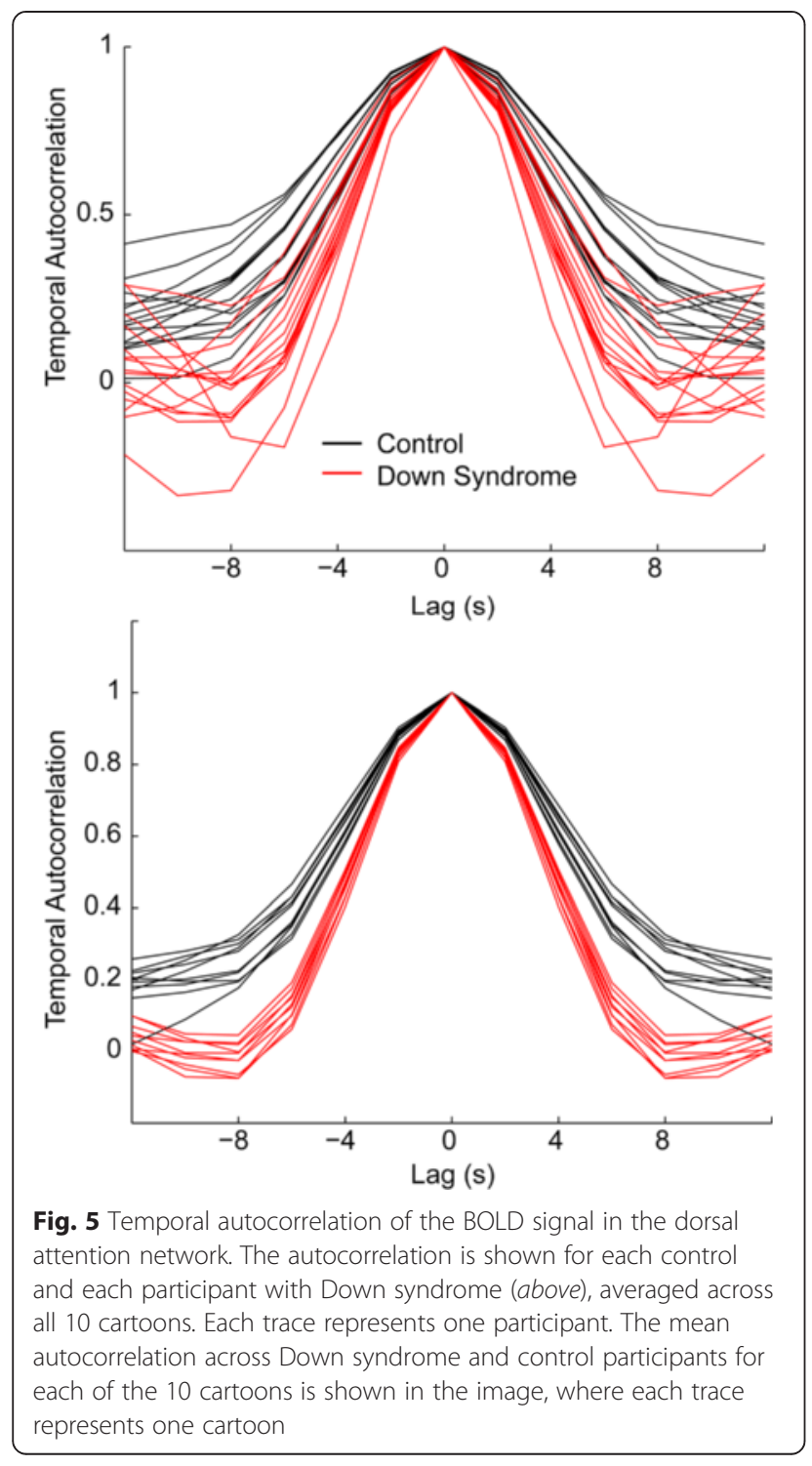

cortex that can be implicated in other neural systems. Nevertheless, these regions are likely to provide a temporal marker for a high-attention state to external audiovisual stimuli.

It is striking that violent scenes predominantly activate this dorsal attention network. Although subjective experiences of the participants in the study cannot be directly inferred from our results, there is provocative evidence that the participants are paying unusual attention to violent scenes. Most of the relative peaks in the traces of Figs. 3 and 4 correspond to violent scenes, and the overall activation map is virtually overlaid with the anatomical boundaries of the dorsal attention network. The strong attentional focus on violent scenes among control individuals likely accentuates differences in attentional response in Down syndrome that may not be specific to violence, but in the context of these cartoon stimuli, it

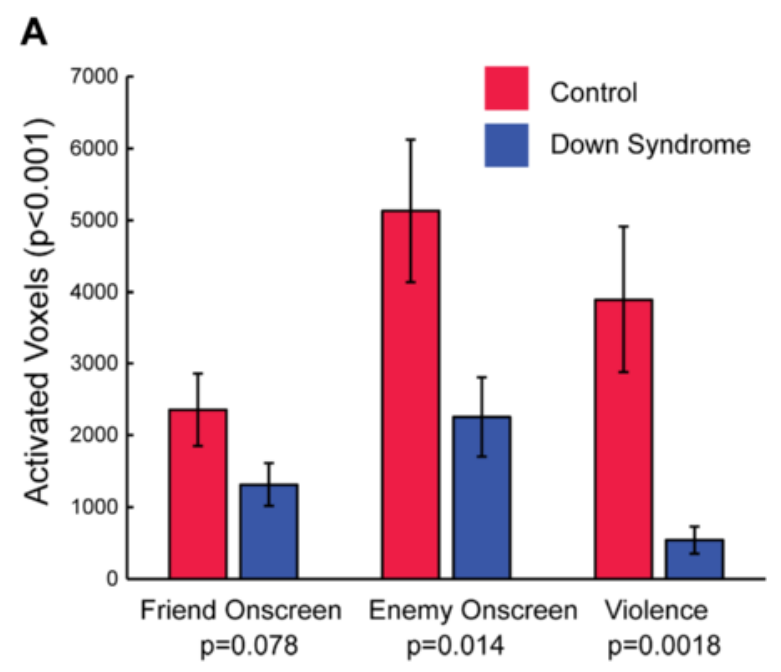

B
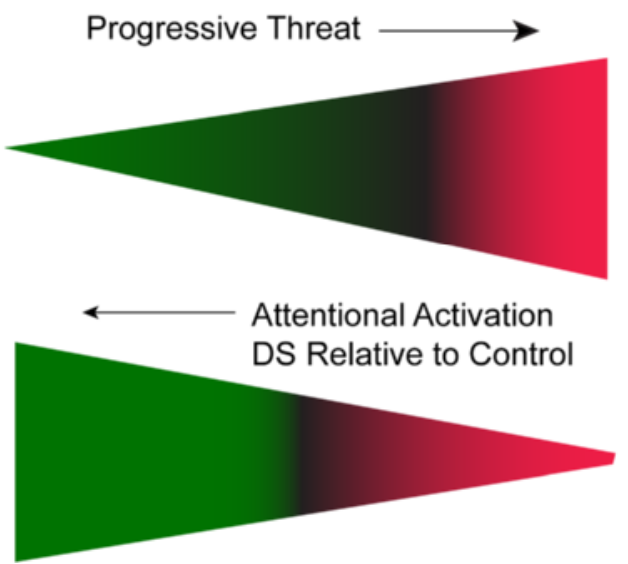

Fig. 6 Brain activation in Down syndrome is specifically weaker as stimuli are more threatening. a Bar graph shows number of activated voxels for $p<0.001$, uncorrected threshold, with error bars showing standard error of the mean across subjects. $P$ values for two-tailed $t$ test between groups are listed for each contrast below the labels. b As relative threat increases from seeing the protagonist to seeing the antagonist to seeing actual violent acts, the relative brain activation in individuals with Down syndrome compared to controls becomes smaller. For all three tasks, activated clusters are predominantly located in association cortex attentional regions

illustrate how generalized inattention in ecological social contexts may be most salient in relation to such emotively powerful events.

Prior reports of brain activation in response to media violence implicate several brain regions that may be more active during violent media viewing or associated with recent or chronic exposure to violent media. In one report of eight children, brain activation during viewing of a boxing scene from Rocky IV compared to viewing a nonviolent clip was greater in the right versus left precuneus, hippocampus, amygdala, and premotor cortex [24]. Another study evaluating responses to violence designed 
to evaluate desensitization overtime found activation to aggressive media scenes in bilateral orbitofrontal cortex, inferior frontal gyrus, anterior and posterior cingulated cortex, bilateral middle temporal, and bilateral middle occipital gyri [25].

In individuals with high exposure to media violence [26] and recent participation in violent video games, [27] activation to a Stroop task was differentially weaker in frontal lobe regions. Avid game players of violent video games exhibited decreased left lateral orbitofrontal activity in response to unpleasant visual stimuli compared to controls in one study [28]. Similarly, individuals with chronic high exposure to media violence exhibited weaker activation to violent images in the right lateral orbitofrontal cortex in another study [29]. In a placebocontrolled study in which participants played a violent video game after administration of quetiapine, an atypical antipsychotic agent, functional connectivity was decreased between the amygdala and the orbitofrontal cortex, with increased connectivity between amygdala and anterior cingulate and dorsolateral prefrontal cortex [30]. In this study, event-related BOLD signal for violent events was greater in the medial intraparietal sulci (visual attentional regions) [30]. A study analyzing PET responses to media violence in individuals with aggressive traits demonstrated decreased glucose metabolism in the medial orbitofrontal cortex for both violent and neutral stimuli [31]. Nevertheless, the brain imaging literature is virtually silent with respect to differences in brain responses to media violence associated with neurodevelopmental conditions. No literature was identified specifically evaluating how the brain is activated by stylized violence such as what is found in violent cartoons.

Medial temporal abnormalities in response to threat in Down syndrome are consistent with neuropsychological testing showing particular dysfunction associated with metrics requiring hippocampal and parahippocampal function [32]. This finding also reinforces approaches to investigation of treatments targeting medial temporal lobe function and supports findings of medial temporal dysfunction in a mouse model of Down syndrome [33]. We note that the medial temporal lobes process many functions that may contribute towards complex emotive responses including emotive salience, memory recall, theory of mind, and language. If the medial temporal lobe is relatively less engaged in responses to threats in Down syndrome, this may have profound effects throughout development on perception of risks of aversive situations, as well as the development of typical emotive responses to threat or impaired recall of semantic memories associated with a potential threat. Given that the brains of individuals with Down syndrome show particularly weak connectivity between distant brain regions, this may indicate a failure to integrate temporal lobe structures with distributed brain networks.

In addition, the failure to activate distributed brain attentional networks in response to threat may result from fundamental impairments in perception observed in visual and auditory cortex. Such primary perceptual deficits are not commonly observed in the literature for other neurodevelopmental disorders such as autism [34] and may represent a more profound functional brain disorder in Down syndrome consistent with the low IQ and more severe impairment of the individuals with Down syndrome in our study. Core perceptual abnormalities may be compounded for more complex attentional and cognitive processes that rely on perception. It seems likely that inattention may be a direct downstream consequence of perceptual activation and that the selective inattention to violence may be a consequence of the fact that violent scenes represent peak attentional events in control individuals. Additionally, attention to violence is a complex concept, integrating not only perception but language, memory, and theory of mind, all of which may be impaired in Down syndrome [35-37]. Other similarly complex social and behavioral interactions may exhibit similar accentuated inattention in Down syndrome, as evidenced by the poor attention to the two romantically themed episodes in our stimuli.

A failure of network-level integration may also be inferred from the reduced temporal autocorrelation in the dorsal attention network. Given that attentional network activation requires communication from disparate sets of loci throughout the brain, poor synchrony of these regions may result in an inability to sustain activations associated with conscious perception. Nevertheless, this possibility will require confirmation with more direct correlates of conscious perception, including subjective reports of experience or validation from other methods, since fMRI activation patterns do not unambiguously connote subjective experience and may be explained by other potential artifacts such as variable hemodynamic coupling or vascular differences. It is also possible that participants with Down syndrome systematically participated in the cartoon task differentially and that findings represent an epiphenomenon of participant involvement rather than abnormalities in brain network architecture. The participants with Down syndrome were relatively low functioning (many with IQ around 40, max full scale IQ 54 ), and the findings may also represent a nonspecific marker of low cognitive function.

It is intriguing that although decreased activation is seen for violent scenes and for scenes featuring the antagonist, participants with Down syndrome show no significant decreases in brain activation in response to Bugs Bunny. This may be consistent with the relatively pro-social features of Down syndrome personality spectra and reinforce 
the hypothesis that positive emotional stimuli may still engage attention networks despite intellectual dysfunction across multiple cognitive domains [38].

Additional studies may help to determine the range of experience over which individuals with Down syndrome may show medial temporal hypoactivation or decreased attention to threatening or violent stimuli. It is possible that our control cohort, with slightly higher IQ than the general population may limit external generalizability of our results. Because the dorsal attention network is defined in typically developing individuals, it is possible that subtle variation in the position or architecture of attentional processes in Down syndrome may underlie observed differences rather than behavioral responses. Nevertheless, Fig. 1 illustrates that the responses in the dorsal attention network appear spatially identical between groups, and similarly, the fact that cartoon viewers appear to show particular attention to violent scenes suggests that more evidence is required to understand the neurodevelopmental impact of violent media viewing. Nevertheless, it may be naïve in light of these results to assert that children or adults watching violent cartoons ignore or dismiss violent imagery.

\section{Conclusions}

Typically developing adolescents and adults demonstrated strong, specific activation of the brain's dorsal attention work in response to violent scenes in animated cartoons. In contrast, dorsal attention network responses in a sample of adolescents and young adults with Down syndrome showed significantly reduced activation in response to violent events during passive cartoon viewing compared to age-matched controls. Brain responses to the presence of an enemy or antagonist showed the greatest reduction within the dorsal attention network and left medial temporal lobe. Hypoactivation of both auditory and visual cortex was observed in response to cartoon viewing in Down syndrome. Finally, brain activation within the dorsal attention network showed reduced autocorrelation in Down syndrome, possibly representing a brain biomarker for reduced attention span in Down syndrome.

\section{Additional file}

Additional file 1: Scenes where DS and control dorsal attention network activity differed. Table S1. Cartoon scenes showing greater relative signal in the dorsal attention network for the Down syndrome sample than for the control sample. $P$ value is calculated for two-tailed $t$ test between subjects. Time is given in seconds from the onset of the cartoon. For scenes lasting longer than $2 \mathrm{~s}$, the peak difference timepoint is shown in parentheses under Mean DS Signal. Table S2. Cartoon scenes showing greater relative signal in the dorsal attention network for the control sample than for the Down syndrome sample. $P$ value is calculated for two-tailed $t$ test between subjects. Time is given in seconds from the onset of the cartoon. For scenes lasting longer than $2 \mathrm{~s}$, the peak difference timepoint is shown in parentheses under Mean DS Signal.

\section{Competing interests}

The authors declare that they have no competing interests.

\section{Authors' contributions}

JSA and JRK participated in conception, design, data acquisition, data analysis, and drafting of the manuscript. SMT participated in data analysis and drafting the manuscript. MAF and JAN participated in data acquisition and data analysis. JOE participated in data acquisition, data analysis, and drafting the manuscript. LD and GG participated in design, data acquisition, and data analysis. All authors approved the final version of the manuscript.

\section{Acknowledgements}

The project described was supported by NIH grant numbers R01HD67731 and K08MH092697. Investigators and funding sources who contributed to the 1000 Functional Connectomes Dataset and ADHD 200 Dataset are available at http://fcon_1000.projects.nitrc.org. The content is solely the responsibility of the authors and does not necessarily represent the official views of the National Institute of Mental Health or the National Institutes of Health.

\section{Author details}

'Department of Radiology, 1 A71 School of Medicine, University of Utah, Salt Lake City, UT 84132, USA. ${ }^{2}$ Interdepartmental Program in Neuroscience, University of Utah, Salt Lake City, USA. ${ }^{3}$ The Brain Institute at the University of Utah, Salt Lake City, USA. ${ }^{4}$ Department of Bioengineering, University of Utah, Salt Lake City, USA. ${ }^{5}$ Department of Psychology, University of Arizona, Tucson, USA. ${ }^{6}$ Department of Pediatrics, University of Utah, Salt Lake City, USA. ${ }^{7}$ Scientific Computing and Imaging Institute, University of Utah, Salt Lake City, USA.

Received: 26 November 2014 Accepted: 26 May 2015 Published online: 03 June 2015

\section{References}

1. Shin M, Besser LM, Kucik JE, Lu C, Siffel C, Correa A. Prevalence of Down syndrome among children and adolescents in 10 regions of the United States. Pediatrics. 2009;124(6):1565-71.

2. Chapman RS, Hesketh LJ. Behavioral phenotype of individuals with Down syndrome. Ment Retard Dev Disabil Res Rev. 2000;6(2):84-95.

3. Gilmore L, Campbell J, Cuskelly M. Developmental expectations, personality stereotypes, and attitudes towards inclusive education: community and teacher views of Down syndrome. Int J Disabil Dev Educ. 2010;50(1):65-76.

4. Laing E, Butterworth G, Ansari D, Gsodl M, Longhi E, Panagiotaki G, et al. Atypical development of language and social communication in toddlers with Williams syndrome. Dev Sci. 2002;5(2):233-46.

5. Wishart JG, Pitcairn TK. Recognition of identity and expression in faces by children with Down syndrome. Am J Ment Retard. 2000;105(6):466-79.

6. Losin EA, Rivera SM, O'Hare ED, Sowell ER, Pinter JD. Abnormal fMR activation pattern during story listening in individuals with Down syndrome. Am J Intellect Dev Disabil. 2009;114(5):369-80.

7. Jacola LM, Byars AW, Chalfonte-Evans M, Schmithorst VJ, Hickey F, Patterson $B$, et al. Functional magnetic resonance imaging of cognitive processing in young adults with Down syndrome. Am J Intellect Dev Disabil. 2011;116(5):344-59.

8. Anderson JS, Nielsen JA, Ferguson MA, Burback MC, Cox ET, Dai L, et al. Abnormal brain synchrony in Down syndrome. Neurolmage. 2013;2:703-15.

9. Vega JN, Hohman TJ, Pryweller JR, Dykens EM, Thornton-Wells TA. Resting state functional connectivity in individuals with Down syndrome and Williams syndrome compared to typically developing controls. Brain Connect. 2015. Ahead of print. doi:10.1089/brain.2014.0266.

10. Pujol J, Del Hoyo L, Blanco-Hinojo L, de Sola S, Macia D, Martinez-Vilavella G, et al. Anomalous brain functional connectivity contributing to poor adaptive behavior in Down syndrome. Cortex. 2015;64:148-56.

11. Imai M, Watanabe H, Yasui K, Kimura Y, Shitara Y, Tsuchida S, et al. Functional connectivity of the cortex of term and preterm infants and infants with Down's syndrome. Neuroimage. 2014;85(Pt 1):272-8.

12. American Psychological Association. Diagnostic and Statistical Manual of Mental Disorders. 5th ed. Washington DC: American Psychological Association; 2013.

13. Kaufman AS. Kaufman Brief Intelligence Test: KBIT. Circle Pines, MN: American Guidance Service; 1990 
14. Biswal BB, Mennes M, Zuo XN, Gohel S, Kelly C, Smith SM, et al. Toward discovery science of human brain function. Proc Natl Acad Sci U S A. 2010;107(10):4734-9.

15. ADHD-200_Consortium. The ADHD-200 Consortium: a model to advance the translational potential of neuroimaging in clinical neuroscience. Front Syst Neurosci. 2012;6:62.

16. Nielsen JA, Zielinski BA, Ferguson MA, Lainhart JE, Anderson JS. An evaluation of the left-brain vs. right-brain hypothesis with resting state functional connectivity magnetic resonance imaging. PLoS One. 2013;8(8), e71275.

17. Anderson JS, Ferguson MA, Lopez-Larson M, Yurgelun-Todd D. Reproducibility of single-subject functional connectivity measurements. AJNR Am J Neuroradiol. 2011;32(3):548-55

18. Yeo BT, Krienen FM, Sepulcre J, Sabuncu MR, Lashkari D, Hollinshead M, et al. The organization of the human cerebral cortex estimated by intrinsic functional connectivity. J Neurophysiol. 2011;106(3):1125-65.

19. Corbetta M, Shulman GL. Control of goal-directed and stimulus-driven attention in the brain. Nat Rev Neurosci. 2002;3(3):201-15.

20. Fox MD, Corbetta M, Snyder AZ, Vincent JL, Raichle ME. Spontaneous neuronal activity distinguishes human dorsal and ventral attention systems. Proc Natl Acad Sci U S A. 2006;103(26):10046-51.

21. Anderson JS, Ferguson MA, Lopez-Larson M, Yurgelun-Todd D. Topographic maps of multisensory attention. Proc Natl Acad Sci U S A 2010;107(46):20110-4.

22. Fox MD, Snyder AZ, Vincent $J$, Corbetta M, Van Essen DC, Raichle ME. The human brain is intrinsically organized into dynamic, anticorrelated functional networks. Proc Natl Acad Sci U S A. 2005;102(27):9673-8.

23. Anderson JS, Ferguson MA, Lopez-Larson M, Yurgelun-Todd D. Connectivity gradients between the default mode and attention control networks. Brain Connect. 2011;1(2):147-57.

24. Murray J, Liotti M, Ingmundson P, Mayberg H, Pu Y, Zamarripa F, et al. Children's brain activations while viewing televised violence revealed by fMRI. Media Pyschol. 2006:8:25-37.

25. Strenziok M, Krueger F, Deshpande G, Lenroot RK, van der Meer E, Grafman J. Fronto-parietal regulation of media violence exposure in adolescents: a multi-method study. Soc Cogn Affect Neurosci. 2011;6(5):537-47.

26. Mathews VP, Kronenberger WG, Wang Y, Lurito JT, Lowe MJ, Dunn DW. Media violence exposure and frontal lobe activation measured by functional magnetic resonance imaging in aggressive and nonaggressive adolescents. J Comput Assist Tomogr. 2005;29(3):287-92.

27. Wang Y, Mathews VP, Kalnin AJ, Mosier KM, Dunn DW, Saykin AJ, et al. Short term exposure to a violent video game induces changes in frontolimbic circuitry in adolescents. Brain Imaging Behav. 2009:3(1):38-50.

28. Montag C, Weber B, Trautner P, Newport B, Markett S, Walter NT, et al. Does excessive play of violent first-person-shooter-video-games dampen brain activity in response to emotional stimuli? Biol Psychol. 2012;89(1):107-11.

29. Kelly CR, Grinband J, Hirsch J. Repeated exposure to media violence is associated with diminished response in an inhibitory frontolimbic network. PLoS One. 2007;2(12), e1268.

30. Klasen M, Zvyagintsev M, Schwenzer M, Mathiak KA, Sarkheil P, Weber R et al. Quetiapine modulates functional connectivity in brain aggression networks. Neuroimage. 2013;75C:20-6.

31. Alia-Klein N, Wang GJ, Preston-Campbell RN, Moeller SJ, Parvaz MA, Zhu W et al. Reactions to media violence: it's in the brain of the beholder. PLoS One. 2014;9(9), e107260.

32. Pennington BF, Moon J, Edgin J, Stedron J, Nadel L. The neuropsychology of Down syndrome: evidence for hippocampal dysfunction. Child Dev. 2003;74(1):75-93.

33. Hanson JE, Blank M, Valenzuela RA, Garner CC, Madison DV. The functional nature of synaptic circuitry is altered in area CA3 of the hippocampus in a mouse model of Down's syndrome. J Physiol. 2007;579(Pt 1):53-67.

34. Anderson JS, Ferguson MA, Nielsen JA. Functional MRI in autism. In: Casanova MF, El-Baz AS, Suri JS, editors. Imaging the brain in autism. New York: Springer; 2013. p. 289-323.

35. Hahn LJ, Fidler DJ, Hepburn SL, Rogers SJ. Early intersubjective skills and the understanding of intentionality in young children with Down syndrome. Res Dev Disabil. 2013;34(12):4455-65.

36. Jarrold C, Baddeley AD, Phillips C. Down syndrome and the phonological loop: the evidence for, and importance of, a specific verbal short-term memory deficit. Downs Syndr Res Pract. 1999;6(2):61-75.
37. Yirmiya N, Solomonica-Levi D, Shulman C, Pilowsky T. Theory of mind abilities in individuals with autism, Down syndrome, and mental retardation of unknown etiology: the role of age and intelligence. J Child Psychol Psychiatry. 1996;37(8):1003-14.

38. Edgin JO, Mason GM, Allman MJ, Capone GT, Deleon I, Maslen C, et al. Development and validation of the Arizona cognitive test battery for Down syndrome. J Neurodev Disord. 2010;2(3):149-64.

\section{Submit your next manuscript to BioMed Central and take full advantage of:}

- Convenient online submission

- Thorough peer review

- No space constraints or color figure charges

- Immediate publication on acceptance

- Inclusion in PubMed, CAS, Scopus and Google Scholar

- Research which is freely available for redistribution 